\title{
Using radial velocities to reveal black holes in binaries: A test case ${ }^{\star}$
}

\author{
M. Clavel ${ }^{1}$, G. Dubus ${ }^{1}$, J. Casares ${ }^{2,3}$, and C. Babusiaux ${ }^{1}$ \\ ${ }^{1}$ Univ. Grenoble Alpes, CNRS, IPAG, 38000 Grenoble, France \\ e-mail: maica.clavel@univ-grenoble-alpes.fr \\ 2 Instituto de Astrofísica de Canarias, 38205 La Laguna, Tenerife, Spain \\ 3 Departamento de Astrofísica, Universidad de La Laguna, 38206 La Laguna, Tenerife, Spain
}

Received 1 September 2020 / Accepted 1 November 2020

\begin{abstract}
Aims. Large radial velocity variations in the LAMOST spectra of giant stars have been used to infer the presence of unseen companions. Some of them have been proposed as possible black hole candidates. We test this selection by investigating the classification of the one candidate that has a known X-ray counterpart (UCAC4 721-037069).

Methods. We obtained time-resolved spectra from the Liverpool Telescope and a 5 ks observation from the Chandra observatory to fully constrain the orbital parameters and the X-ray emission of this system.

Results. We find the source to be an eclipsing stellar binary that can be classified as an RS CVn. The giant star fills its Roche lobe, and the binary mass ratio is greater than one. The system may be an example of stable mass transfer from an intermediate-mass star with a convective envelope.

Conclusions. Using only radial velocity to identify black hole candidates can lead to many false positives. The presence of an optical orbital modulation, such as what has been observed for all LAMOST candidates, will in most cases indicate that the system is a stellar binary.
\end{abstract}

Key words. stars: black holes - stars: individual: UCAC4 721-037069 - X-rays: stars - binaries: eclipsing techniques: radial velocities

\section{Introduction}

Known compact binaries with neutron stars (NSs) or black holes (BHs) as compact objects are currently identified thanks to their bright X-ray outbursts (McClintock \& Remillard 2006; Corral-Santana et al. 2016) or from follow-up observations of X-ray surveys (e.g., Walter et al. 2015). However, these bright $\mathrm{X}$-ray sources most likely represent only a small fraction of the overall population of compact binaries, with a larger number of $\mathrm{X}$-ray faint objects missed by the current approach because of long quiescent periods in between outbursts (Dubus et al. 2001; Yungelson et al. 2006; Yan \& Yu 2015) or because they are persistently very faint (Menou et al. 1999; King \& Wijnands 2006).

Radial velocity variations in optical spectra could be a means to uncover the population of quiescent compact objects that have a stellar companion (Casares et al. 2014; Giesers et al. 2018; Thompson et al. 2019; Makarov \& Tokovinin 2019). Recently, $\mathrm{Gu}$ et al. (2019) selected spectra of bright late-type giants obtained at different times by the LAMOST spectral survey and identified seven candidates with large radial velocity variations that are compatible with the presence of an unseen solar mass companion of $>3 M_{\odot}$. One of them (UCAC4 721-037069, which corresponds to their source \#4, hereafter GS4) has a possible $\mathrm{X}$-ray counterpart and is presented by Gu et al. (2019) as their best $\mathrm{BH}$ candidate.

\footnotetext{
* Radial velocities are only available at the CDS via anonymous ftp to cdsarc.u-strasbg. fr (130.79.128.5) or via http://cdsarc. u-strasbg.fr/viz-bin/cat/J/A+A/645/A72
}

In this paper, we investigate the available data, including two dedicated observation programs, to identify the nature of this intriguing source. The optical spectra, the orbital parameters, and the properties of the X-ray counterpart of GS4 are described in Sect. 2. The identification of this source as an RS $\mathrm{CVn}$ is then discussed in Sect. 3, along with considerations on how constraints on the orbital period could further help discriminate between this type of source and quiescent BHs that have a stellar companion.

\section{Optical and X-ray constraints}

GS4 corresponds to Gaia DR2 273187064220377600. Located at RA $(\mathrm{J} 2000)=04^{\mathrm{h}} 56^{\mathrm{m}} 12.7739^{\mathrm{s}}$ and Dec $(\mathrm{J} 2000)=54^{\circ} 00^{\prime} 21$. $2768^{\prime \prime}$, this source has a parallax of $1.07 \pm 0.03$ mas, which corresponds to a distance of $0.91 \pm 0.03 \mathrm{kpc}$ (Bailer-Jones et al. 2018).

The All-Sky Automated Survey for Supernovae (ASASSN, Shappee et al. 2014; Kochanek et al. 2017; Jayasinghe et al. 2019) and Gaia DR2 (Gaia Collaboration 2016, 2018) provide aperture photometry light curves for GS4. As previously reported by Zheng et al. (2019), these light curves have a strong modulation with a period $p \approx 5.2$ days, and the morphology of GS4's folded light curve is typical of eclipsing binaries (see Fig. 1, top, and Sect. 2.2).

The extinction toward GS4 can be estimated from the 3D map of interstellar dust reddening derived from the PanSTARRS 1 and 2MASS surveys (Green et al. 2018). At $910 \mathrm{pc}$, the reddening is $E(g-r)=0.35 \pm 0.02$, which corresponds to an 
extinction $A_{V}=1.06 \pm 0.06^{1}$. This value converts into a column density $N_{\mathrm{H}} \approx 2.3 \times 10^{21} \mathrm{~cm}^{-2}$ (see Güver \& Özel 2009).

\subsection{Time-resolved spectroscopy and radial velocity}

In agreement with the star's position in the Gaia HertzsprungRussell diagram, the LAMOST spectra of GS4 reveal a late-type giant that has radial velocity variations as large as $\Delta V_{\mathrm{r}}=127.2 \pm$ $7.8 \mathrm{~km} \mathrm{~s}^{-1}$ (Gu et al. 2019; Zheng et al. 2019). We obtained refined radial velocity measurements using the medium resolution spectrograph FRODOSpec at the robotic Liverpool Telescope (LT, Steele et al. 2004; Barnsley et al. 2012) to establish the orbital parameters of this source.

The spectrograph is fed by a fiber bundle array consisting of $12 \times 12$ lenslets of $0.82^{\prime \prime}$ each, which is reformatted as a slit. FRODOSpec was operated in high resolution mode, providing a spectral resolving power of $R \sim 5500$ in the blue arm and $R \sim 5300$ in the red arm. The spectral coverage was 3900-5215 $\AA$ and 5900-8000 A, respectively. A total of 26 spectra of $1200 \mathrm{~s}$ were obtained in each arm, with a cadence of 1-3 spectra per night between 2019 August 28 and 2019 December 1 . For the sake of radial velocity analysis, we also obtained a $300 \mathrm{~s}$ spectrum of the K0III standard HD 210185 on the night of 2019 September 17, using the same spectral configuration. The FRODOSpec pipeline produces fully extracted and wavelengthcalibrated spectra with rms $\leq 0.1 \AA$ above $4400 \AA$. The analysis presented in this paper was performed with the FRODOSpec pipeline products.

Radial velocities were extracted through the crosscorrelation of every GS4 red-arm spectrum with the K0III template in the spectral range 6300-7900 $\AA$, after masking the main telluric and interstellar absorption bands. The $\mathrm{H} \alpha$ line was also excluded as some spectra show evidence of variable narrowemission, reminiscent of chromospheric activity.

The spectrum obtained for GS4 is compatible with a K0III star that has a temperature on the order of $4800 \mathrm{~K}$ (consistent with the effective temperature derived from LAMOST DR6, see e.g., Gu et al. 2019), and a periodogram analysis of the radial velocity points shows a clear peak at 5.2081(34)d, in excellent agreement with the ASAS-SN photometric period (see below). The radial velocity curve is shown in Fig. 1 (bottom). Modeling the light curve with a simple sinusoid gives the systemic velocity $\gamma=-7.8 \pm 1.1 \mathrm{~km} \mathrm{~s}^{-1}$ and the semi-amplitude radial velocity $K_{1}=69.0 \pm 1.7 \mathrm{~km} \mathrm{~s}^{-1}$. Together with the orbital period of the system determined by phase dispersion minimization on the ASAS-SN $V$-band light curve, $P_{\text {orb }}=5.20906 \pm 0.0005 \mathrm{~d}$, this corresponds to a binary mass function $f=0.18 \pm 0.01 M_{\odot}$.

We observe a shift of 0.04 in phase between the ASAS-SN light curve and the LT radial velocity curve (see Fig. 1, bottom). This corresponds to five hours, so we can rule out any instrumental effect ${ }^{2}$. A persistent asymmetry in the stellar radiation field (due to, e.g., star spots, see also Sect. 3.1) could be a possible physical origin, but we have no evidence for this. We note that an asymmetric flux distribution combined with eclipses

\footnotetext{
1 Using the Gaia and 2MASS surveys, Lallement et al. (2019) derived an extinction of $1.4 \mathrm{mag}$ in this direction and at this distance. Using this value would require a higher luminosity from the binary components to account for the observed flux: It would increase the tension between spectral type and luminosity, furthering the need for additional light (see Sects. 2.2 and 3.1).

2 Both the LT and ASAS-SN curves are provided in Heliocentric Julian Day (HJD), and the ASAS-SN photometry aligns well with publicly available data from other observatories (e.g., Gaia).
}

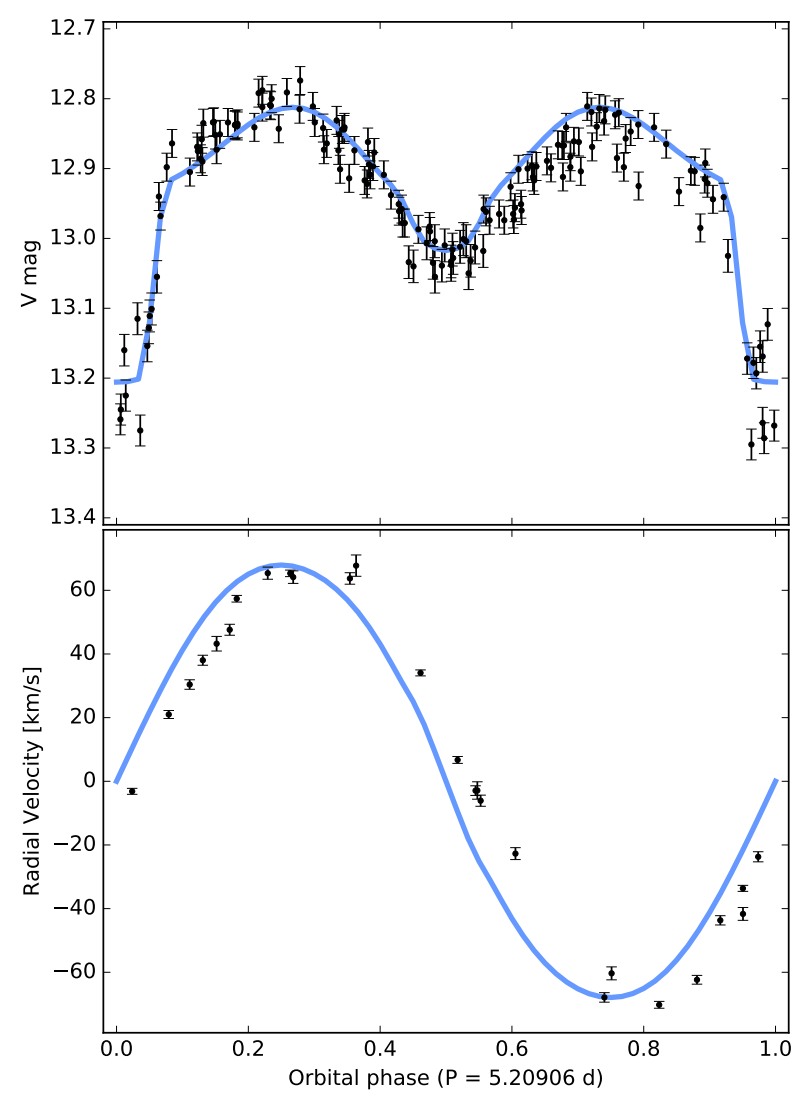

Fig. 1. Aperture photometry and radial velocity. Top: ASAS-SN light curve of GS4, folded using the period $p=5.20906 \pm 0.0005 \mathrm{~d}$ and $t_{0}=2457384.6$ HJD. Bottom: corresponding radial velocity of the system as observed by the LT. We do see a 0.04 phase shift between the radial velocity curve and the ASAS-SN light curve, but we have no clear explanation for it (see Sect. 2.1). The blue curves correspond to the best fit model obtained with PHOEBE on the ASAS-SN light curve when fixing the semi-amplitude radial velocity of the system to the one measured from the LT curve (see Sect. 2.2).

has also been discussed as the possible origin of a similar phase lag detected in the BH X-ray binary XTE J1118+480 during its decay to quiescence (McClintock et al. 2001).

In order to constrain the rotational broadening of the KOIII star, we broadened the red-arm spectrum of the template star (HD 210185) from 0 to $120 \mathrm{~km} \mathrm{~s}^{-1}$ in steps of $2 \mathrm{~km} \mathrm{~s}^{-1}$ using a Gray profile (Gray 1992) with a linear limb-darkening law with coefficient $\epsilon=0.75$, which is appropriate for the wavelength range and spectral type of our star (see Al-Naimiy 1978). The broadened versions of the template star were multiplied by fractions $f<1$ to account for the fractional contribution to the total light and, subsequently, subtracted from the Doppler-corrected average of the 26 red spectra of GS4 using our orbital solution. A $\chi^{2}$ test on the residuals yields $V \sin i=80 \pm 1 \mathrm{~km} \mathrm{~s}^{-1}$. The comparison of the GS4 spectrum with the best broadened version of the template star is shown in Fig. 2 (top).

\subsection{System parameters derived from the light curve}

We modeled the ASAS-SN light curve using the eclipsing binary modeling software PHOEBE ${ }^{3}$ with PHOENIX model atmosphere (Jones et al. 2020), in combination with the Markov chain Monte Carlo (MCMC) sampler emcee (Foreman-Mackey et al. 2013). We fixed the orbital period to the value derived from

\footnotetext{
3 http://phoebe-project.org/
} 
M. Clavel et al.: Using radial velocities to reveal black holes in binaries: A test case
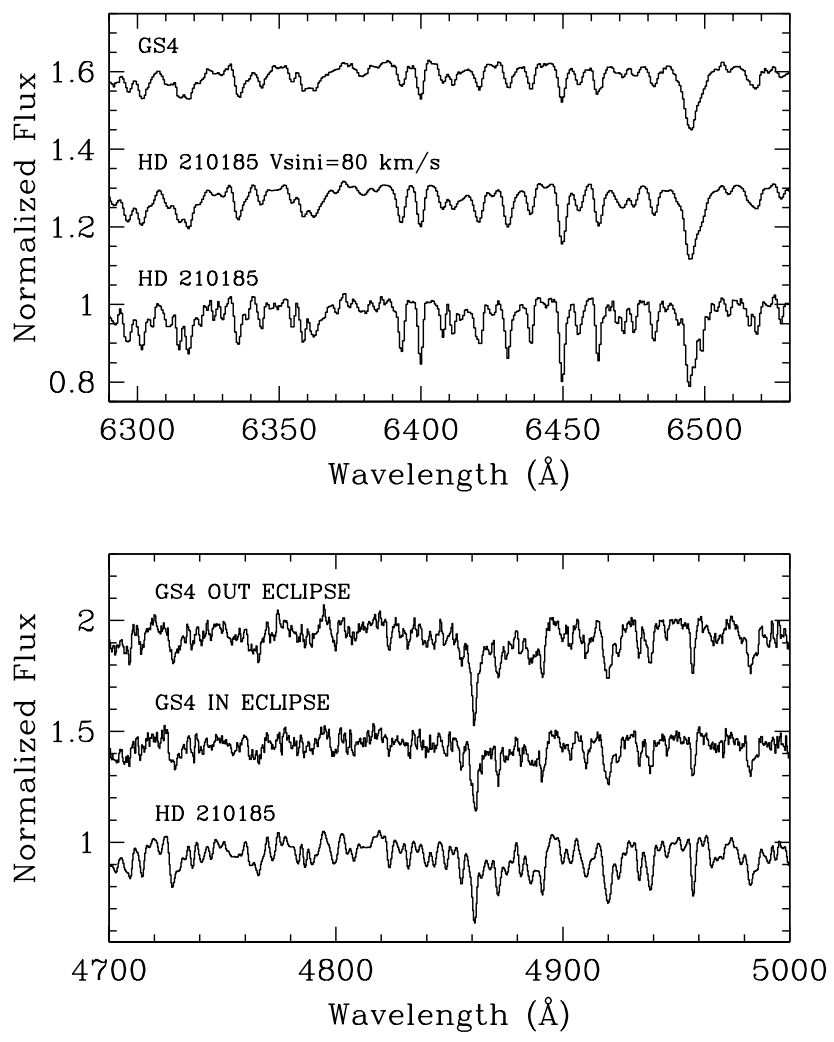

Fig. 2. Sample of LT spectra. Top: from bottom to top: red-arm LT spectrum of HD 210185 (the K0III template), the same spectrum broadened by $80 \mathrm{~km} \mathrm{~s}^{-1}$, and the Doppler-corrected average spectrum of GS4. Bottom: from bottom to top: blue-arm LT spectrum of the KOIII template in comparison with the Doppler-corrected averages of GS4 obtained during the primary eclipse (photometric phase $0.00 \pm 0.05$ ) and outside the eclipse (remaining phases). The top spectrum shows the $\mathrm{H} \beta$ line (at $4861 \AA$ ) to be slightly stronger (relative to the nearby metallic lines), possibly because of the contribution from the A-type secondary. The secondary is eclipsed in the middle spectrum, which seems to show a better match to the KOIII spectrum. Scaling factors have been applied to the three spectra so that the equivalent widths of the metallic lines in the 4900-5000 A range are the same.

phase dispersion minimization (see Sect. 2.1). We set the reddening to $A_{V}=1.06$, derived from the map of Green et al. (2018, see above). The free parameters of the fit were the system inclination $\cos i$, the primary (K0III) star mass $M_{1}$, the effective temperatures of the binary components $T_{1,2}$, the ratio of the primary star radius $R_{1}$ to the Roche lobe radius $R_{\mathrm{L}}$, the radius of the secondary (unseen) star $R_{2}$, and the parallax $\pi$. The mass of the secondary star $M_{2}$ was set from $M_{1}, i$, and $K_{1}=69 \mathrm{~km} \mathrm{~s}^{-1}$. We used flat priors except for the parallax $\pi$, where we used a Gaussian prior ( $1.0981 \pm 0.0421$ mas). The parallax takes into account the parallax zero point of 0.03 mas and the error increase suggested by Lindegren et al. ${ }^{4}$. The sampler ran for 30000 steps, after which we found that the chain had converged, based on the sampled parameters and the autocorrelation time. We discarded the initial 5000 steps (burn-in phase). The errors quoted in Table 1 are based on the tenth and 90th percentiles. A major source of uncertainty is the adopted reddening. We did not attempt to model light curves in different bands. Taking $A_{V}=0$ significantly lowers the effective temperatures $\left(T_{1} \approx 5060 \mathrm{~K}\right.$ and $T_{2} \approx 7860 \mathrm{~K}$ ), significantly lowers $R_{2}$ to $1.3 R_{\odot}$, and slightly

\footnotetext{
4 https://www. cosmos. esa.int/web/gaia/ dr2-known-issues\#AstrometryConsiderations
}

Table 1. Parameters of the binary system GS4 obtained from a fit of the ASAS-SN $V$-band light curve using PHOEBE.

\begin{tabular}{lcc}
\hline \hline Parameters & Units & Best fit values \\
\hline$i$ & $\circ$ & $78.5_{-0.9}^{+1.2}$ \\
$M_{1}$ & $M_{\odot}$ & $1.9 \pm 0.3$ \\
$M_{2}$ & $M_{\odot}$ & $1.2 \pm 0.1$ \\
$R_{1} / R_{\mathrm{L}}$ & & $0.982_{-0.008}^{+0.005}$ \\
$R_{2}$ & $R_{\odot}$ & $1.7 \pm 0.2$ \\
$T_{1}$ & $\mathrm{~K}$ & $5900_{-150}^{+200}$ \\
$T_{2}$ & $\mathrm{~K}$ & $8800_{-400}^{+500}$ \\
$\pi$ & mas & $1.14 \pm 0.05$ \\
\hline
\end{tabular}

Notes. The errors are based on the tenth and 90th percentiles (see Sect. 2.2). Part of these parameters are likely slightly off compared to the true values due to an extra-light contamination (see Sect. 3.1).

lowers $M_{1}$ to $1.6 M_{\odot}$, but it has no impact on $i$ or $R_{1} / R_{\mathrm{L}}$ since they are constrained by the light curve shape. In all cases, the primary nearly fills its Roche lobe, with $R_{1}=7.5 \pm 0.5 R_{\odot}$.

\subsection{X-ray counterpart}

As pointed out by Gu et al. (2019), GS4 is compatible with the position of the ROSAT source 2RXS J045612.8+540024 (4" away from GS4, with a 12" X-ray position uncertainty, Voges et al. 2000), with an association likelihood of 55\% (Flesch 2016). In the $0.1-2.4 \mathrm{keV}$ range, this $X$-ray source is rather hard, with an X-ray flux of $F_{0.1-2.4 \mathrm{keV}}=4.4 \times 10^{-13} \mathrm{erg} \mathrm{cm}^{-2} \mathrm{~s}^{-1}$. To test this association, we obtained a $5 \mathrm{ks}$ Chandra/ACIS-I observation (Obs. ID 22399) that targets the Gaia position of GS4. This X-ray observation spanned from MJD 58728.017 to 58728.075 , which corresponds to phase $\approx 0.02$ in Fig. 1 : It was performed during the primary eclipse.

The data was reduced using CIAO software v.4.12 along with the calibration database CALDB v.4.9.0. Chandra_repro was used to produce the clean event file, and then wavdetect (default parameters) was run to detect all sources in the field of view. Sixteen sources were detected, including one source consistent with the Gaia position of GS4, detected with a significance of $18 \sigma$. The X-ray source coordinates are RA $=$ $04^{\mathrm{h}} 56^{\mathrm{m}} 12.76^{\mathrm{s}}$, Dec $=54^{\circ} 00^{\prime} 21.2^{\prime \prime}$. The source was therefore named CXO J045612.8+540021. Its position accuracy is limited by the Chandra absolute astrometric accuracy and is therefore $\sim 1^{\prime \prime}$.

We then extracted the source spectrum using specextract and a circular region of radius $r=2^{\prime \prime}$ centered on the position of CXO J045612.8+540021, while the background spectrum was extracted from an annulus region between $5^{\prime \prime}$ and $20^{\prime \prime}$ and centered on the same coordinates. A total of 36 counts were detected from the source in the $0.5-8 \mathrm{keV}$ energy range (there are ten counts in the background region in the same energy range, so we only expect a 0.1 background count in the source region). The source spectrum was binned to have at least five counts per bin and then background subtracted. We note that the last bin from 5 to $8 \mathrm{keV}$ only contains one count. We therefore used Gehrels weights (Gehrels 1986) to better account for the errorbar in each bin. Spectral fits were performed with Xspec v.12.10.1, and the errorbars correspond to the $90 \%$ confidence interval. The absorption is modeled with tbabs, using Wilms abundances (Wilms et al. 2000), and fixed to $N_{\mathrm{H}}=2.3 \times 10^{21} \mathrm{~cm}^{-2}$ (see beginning of Sect. 2). 
We first tested a power-law model to fit the source spectrum. The best fit gives a reduced $\chi_{\mathrm{r}}^{2}=0.4$ (six degrees of freedom, d.o.f.) and a photon index $\Gamma=1.6_{-0.8}^{+1.0}$. The source spectrum is somewhat better fitted by a black body model $\left(\chi_{\mathrm{r}}^{2}=0.3\right.$, six d.o.f.) with a temperature $k T=0.8_{-0.3}^{+0.4} \mathrm{keV}$; the $0.5-10 \mathrm{keV}$ flux of the source is then $F_{0.5-10 \mathrm{keV}}=9.2_{-4.8}^{+5.5} \times 10^{-14} \mathrm{erg} \mathrm{cm}^{-2} \mathrm{~s}^{-1}$, which, at a distance of $0.91 \mathrm{kpc}$, translates into a luminosity $L_{0.5-10 \mathrm{keV}}=(9 \pm 5) \times 10^{30} \mathrm{erg} \mathrm{s}^{-1}$.

Extrapolating the best fit model to the ROSAT energy range, we find a flux that is $F_{0.1-2.4 \mathrm{keV}}=3.4 \times 10^{-14} \mathrm{erg} \mathrm{cm}^{-2} \mathrm{~s}^{-1}$. This is more than one order of magnitude below the ROSAT detection in 1990. While we cannot exclude that the ROSAT source could be a transient that is unrelated to GS4 and that is now under the detection limit of our 2019 Chandra observation, it is also likely that the source has varied, either due to the eclipsing pattern and/or a longer-term variability.

\section{Discussion}

\subsection{Nature of the unseen companion}

The orbital model fitting the orbital light curve of GS4, presented in Sect. 2.2, describes a stellar binary composed of a giant star nearly filling its Roche lobe and a hotter but smaller companion. At phase 0 , the giant star is totally eclipsing its companion, while at phase 0.5 the companion is in front of the giant star and only partly eclipsing it. The deformed shape of the giant star produces the ellipsoidal modulation in addition to the eclipse at phase 0 . Therefore, the fit to the orbital modulation constrains the inclination and the Roche lobe filling factor of the primary very well. The rotational broadening $\left(\approx 80 \mathrm{~km} \mathrm{~s}^{-1}\right)$ is compatible with the primary star being tidally locked and synchronized with $R_{1} \approx 8 R_{\odot}$, as expected since it nearly fills its Roche lobe.

Under the assumption that the K0III star fully fills its Roche lobe and is tidally locked in its orbit, the binary mass ratio $q$ can be derived through the expression $V \sin i \simeq 0.462 K_{1} q^{1 / 3}(1+q)^{2 / 3}$ (Wade \& Horne 1988). In our case, $q=M_{1} / M_{2}$, where $M_{1}$ is the mass of the K0III star and $K_{1}=69.0 \pm 1.7 \mathrm{~km} \mathrm{~s}^{-1}$, so we find $q=1.89 \pm 0.07$. This is slightly larger than, but consistent with, the mass ratio derived through the PHOEBE modeling (Table 1).

From the orbital evolution of the veiling factor (i.e., the fractional contribution of the KOIII template spectrum to the total light), we find that the K0III spectrum contributes $60 \%$ outside the primary eclipse and $91 \%$ at phase 0 . This means that the secondary accounts for $\approx 30 \%$ of the total flux and suggests the presence of additional light in the system that is not accounted for in the orbital modulation fit. Such additional light could also explain why the temperature $T_{1} \approx 5800 \mathrm{~K}$ derived from the fit is incompatible with the K0III spectral classification $(\approx 4800 \mathrm{~K})$. Since the primary star nearly fills its Roche lobe, a mass transfer creating an accretion disk around the secondary star is the most likely explanation for this additional source of light.

The mass, temperature, and radius of the secondary derived from the orbital fit suggest an A- or F-type main sequence companion. This identification of the unseen companion is fully consistent with the fact that it is not detected spectroscopically. It is worth noting that at phase $\sim 0.5$ the spectrum of the secondary is hinted at by the large depth of the Balmer lines (especially $\mathrm{H} \beta$ and $\mathrm{H} \gamma$ in the blue-arm LT spectra) relative to the nearby metallic lines, as compared to a typical K0 spectrum (see Fig. 2, bottom).

These optical constraints and the X-ray emission described in Sect. 2.3 are fully consistent with a chromospherically active giant star in a semidetached binary, and we therefore classify

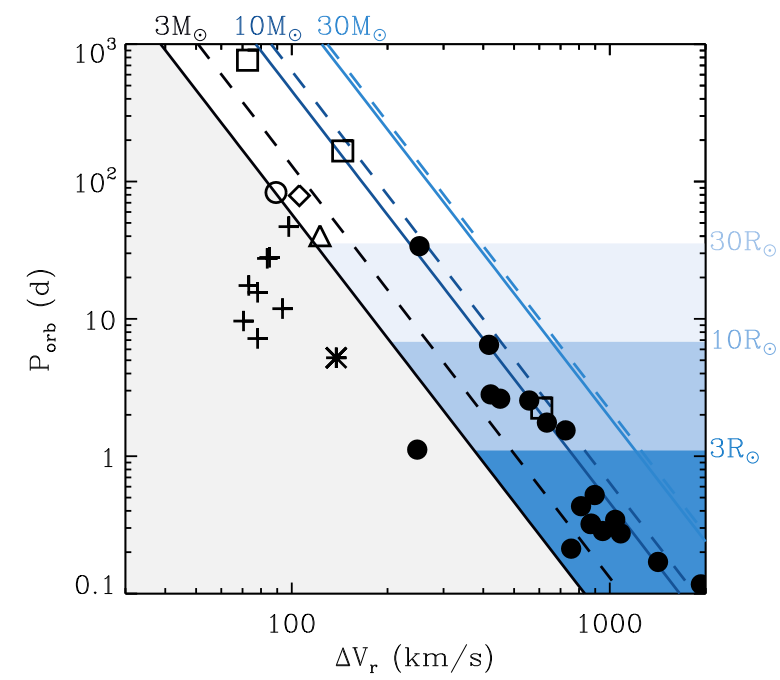

Fig. 3. Black hole candidates with solar mass companions in the orbital period - radial velocity amplitude plane. Solid (dashed) lines show the lower limit on $P_{\text {orb }}$ that, according to Eq. (2), is sufficient to have a 3, 10, or $30 M_{\odot} \mathrm{BH}$ in circular orbit with a $3 M_{\odot}$ stellar companion ( $1 M_{\odot}$ companion). Colored areas indicate where a 3,10 , or $30 R_{\odot}$ stellar companion would overfill its Roche lobe. Candidate $\mathrm{BHs}$ from radial velocity measurements: low-mass X-ray binaries (black dots); APOGEE candidate (open circle); LAMOST LB-1 candidate (open diamond); MUSE candidates in NGC 3201 (open squares); HR 6819 candidate (open triangle) and the latest LAMOST selection of giant stars having large $\Delta V_{\mathrm{r}}$ and optically invisible companions (crosses), including GS4 (star). More details are given in the text.

GS4 as an RS CVn (see e.g., Pandey \& Singh 2012, for the X-ray properties of such systems). These systems do display variability in their X-ray and orbital modulation light curves due to the activity of the giant star. We note that the binary mass ratio is relatively large $(\sim 1.6-1.9)$. This value approaches (but does not exceed) the critical mass ratio for unstable mass transfer from intermediate-mass donor stars with convective envelopes, as computed in recent simulations (Misra et al. 2020). In addition, the asymmetry of the light curve seen at phase $0.8-0.9$ (Fig. 1, top) is fairly typical for an RS CVn and is explained by the presence of starspots at the surface of the giant star.

\subsection{Radial velocities as a probe for quiescent black holes}

Population studies indicate that there may be from $10^{3}$ to $10^{5}$ BHs with solar mass companions (e.g., Shao \& Li 2019; Olejak et al. 2020). The $\approx 60 \mathrm{BH} X$-ray binaries sample only a fraction of this binary population (Corral-Santana et al. 2016). With a duty cycle $\leq 0.02$ (Yan \& Yu 2015), many are hiding in quiescence, with the accretion disk building up mass before the next outburst. Many more systems may be non-accreting if they were unable to evolve to shorter periods during the common envelope phase or through angular momentum losses (de Kool et al. 1987; Iben et al. 1995; Podsiadlowski et al. 2003; Yungelson et al. 2006; Shao \& Li 2019). These binaries can therefore provide important insights into $\mathrm{BH}$ evolution even though they harbor only a small fraction of the $\sim 10^{8}$ BHs estimated to reside in our Galaxy.

Radial velocity surveys of field or globular cluster stars can provide dynamical evidence for invisible companions (Giesers et al. 2018; Zheng et al. 2019; Gu et al. 2019; Yi et al. 2019; Wiktorowicz et al. 2020). For a circular binary, 
$M_{\mathrm{bh}}=\frac{P_{\mathrm{orb}} K_{\star}^{3}(1+q)^{2}}{2 \pi G \sin ^{3} i}$,

where $K_{\star}$ is the semi-amplitude of the radial velocity modulation of the stellar companion and $q=M_{\star} / M_{\mathrm{bh}}$. If a radial velocity difference $\Delta V_{\mathrm{r}}$ is measured between two epochs, a sufficient condition to have a $>3 M_{\odot}$ unseen companion is

$\frac{P_{\text {orb }} \Delta V_{\mathrm{r}}^{3}(1+q)^{2}}{16 \pi G} \geq 3 M_{\odot}$.

We plot this limit in Fig. 3 for $M_{\mathrm{bh}}=3,10$, and $30 M_{\odot}$, assuming $M_{\star}=1$ (dashed lines) or 3 (solid lines) $M_{\odot}$. It is important to highlight that these lines would be shifted toward the left for systems that are known to have a low inclination. The limit below which the star will overfill its Roche lobe is also highlighted. Overlaid on this plot, we show BH candidates based on radial velocity measurements: the $\mathrm{BH}$ low-mass X-ray binaries listed in Table A.4 of Corral-Santana et al. (2016), the BH candidate found in the APOGEE survey (Thompson et al. 2019), the candidate LB-1 found in the LAMOST survey (we note the companion is a B star, Liu et al. 2019, and follow-up discussions by e.g., Irrgang et al. 2020; Shenar et al. 2020), the BH candidates found in MUSE observations of the NGC 3201 globular cluster (some have nonzero eccentricity, Giesers et al. 2019), and the non-accreting BH candidate in the triple system HR 6819 (Rivinius et al. 2020, but see also follow-up discussions in: Bodensteiner et al. 2020; Safarzadeh et al. 2020; El-Badry \& Quataert 2020). X-ray binaries are nearly all located within the mass transfer region and above the limit defined by Eq. (2), except $4 \mathrm{U} 1543-475$, which is known to have a low inclination $i \approx 20^{\circ}$.

For comparison, the latest selection of giant stars that have large $\Delta V_{\mathrm{r}}$ and optically invisible companions from the LAMOST survey (Zheng et al. 2019) are also shown. All, including GS4, lie in the gray area, indicating that the constraint on the radial velocity is not sufficient to reveal quiescent BHs given their relatively short orbital periods. To match the $M_{\mathrm{bh}} \geq 3 M_{\odot}$ criterion, these systems would need to have an upper limit on their inclination of $i \lesssim 65^{\circ}$ for the source closest to the line (source \#8 in Zheng et al. 2019) and between $i \lesssim 40^{\circ}$ and $i \lesssim 20^{\circ}$ for all the other candidates, if we assume the conservative $M_{\star}=3 M_{\odot}$. However, at such a low inclination, we do not expect to detect the strong ellipsoidal modulations that are seen in the ASAS-SN light curves (see e.g., Zheng et al. 2019). Therefore, it is likely that most of these systems are also stellar binaries.

Non-interacting systems clearly lie at longer orbital periods, as supported by the recent detection of one such system using the APOGEE radial velocity survey (Thompson et al. 2019, and follow-up discussions: van den Heuvel \& Tauris 2020; Thompson et al. 2020). A simple, conservative criterion to select candidate BH systems for follow-up is, using Eq. (2),

$\Delta V_{\mathrm{r}} \geq 120\left(\frac{\Delta t}{30 \mathrm{~d}}\right)^{-1 / 3} \mathrm{~km} \mathrm{~s}^{-1}$,

with $\Delta t$ the elapsed time between the maximum and minimum velocity. This is similar to how Thompson et al. (2019) prioritized their candidate list for follow-up, leading to the detection of a strong candidate. GS4, with an initial $\Delta V_{\mathrm{r}}=100 \mathrm{~km} \mathrm{~s}^{-1}$ measured from the survey (Gu et al. 2019), is at the limit of the criterion, although this is possibly lowered by a long $\Delta t$ (the six LAMOST observations are probably spread in time over several years). Surveys with long time bases such as LAMOST are well suited to probe the region of the diagram with detached giant stellar companions. However, as GS4 highlights, the long $\Delta t$ induced by the sampling may also lead to the incorrect identification of stellar binaries with short orbital periods as BH candidates. A work-around to avoid these false positives could be to sample spectra on various timescales.

\section{Conclusion}

The large radial velocity variations detected in LAMOST spectra have been used to highlight potential $\mathrm{BH}$ candidates (Gu et al. 2019; Zheng et al. 2019). We tested this selection by further investigating the parameters of the one system, GS4, that has a known X-ray counterpart and which was presented by Gu et al. (2019) as a likely BH or NS system with mass transfer from the giant to the compact object. The time-resolved spectra that we obtained from the LT along with the ASAS-SN $V$-band light curve of GS4 allowed us to fully constrain the orbital parameters of this eclipsing binary, thereby excluding the possibility of a massive and compact companion. Our Chandra observation did confirm the existence of an X-ray counterpart for this system: its $\mathrm{X}$-ray spectrum and luminosity are fully consistent with a chromospherically active giant star, allowing us to classify GS4 as an RS CVn.

Given the radial velocity variations sampled by LAMOST $\Delta V_{\mathrm{r}} \sim 100 \mathrm{~km} \mathrm{~s}^{-1}$ and the very high inclination of GS4 constrained from its eclipsing behavior, we argue that its short orbital period $P_{\text {orb }} \approx 5.2 \mathrm{~d}$ is sufficient to exclude the BH companion hypothesis for this system. The same reasoning likely also applies to all other LAMOST candidates, except for one (source \#8 in Zheng et al. 2019), since they all have similar radial velocity variations, relatively short orbital periods, and rather high inclinations inferred from the orbital modulations seen in their ASAS-SN light curve (Zheng et al. 2019). Therefore, the orbital period or the timescale of the radial velocity variations appear as the keys to verify the viability of these radial-velocity selected $\mathrm{BH}$ candidates.

Acknowledgements. The scientific results reported in this article are based on observations made with the Liverpool Telescope operated on the island of La Palma by Liverpool John Moores University in the Spanish Observatorio del Roque de los Muchachos of the Instituto de Astrofisica de Canarias with financial support from the UK Science and Technology Facilities Council, and on observations made by the Chandra X-ray Observatory. MC and GD acknowledge financial support from the Centre National d'Etudes Spatiales (CNES). JC acknowledges support by the Spanish MINECO under grant AYA2017-83216-P.

\section{References}

Al-Naimiy, H. M. 1978, Ap\&SS, 53, 181

Bailer-Jones, C. A. L., Rybizki, J., Fouesneau, M., Mantelet, G., \& Andrae, R. 2018, AJ, 156, 58

Barnsley, R. M., Smith, R. J., \& Steele, I. A. 2012, Astron. Nachr., 333, 101

Bodensteiner, J., Shenar, T., Mahy, L., et al. 2020, A\&A, 641, A43

Casares, J., Negueruela, I., Ribó, M., et al. 2014, Nature, 505, 378

Corral-Santana, J. M., Casares, J., Muñoz-Darias, T., et al. 2016, A\&A, 587, A61

de Kool, M., van den Heuvel, E. P. J., \& Pylyser, E. 1987, A\&A, 183, 47

Dubus, G., Hameury, J. M., \& Lasota, J. P. 2001, A\&A, 373, 251

El-Badry, K., \& Quataert, E. 2020, MNRAS, submitted [arXiv:2006.11974]

Flesch, E. W. 2016, PASA, 33, e052

Foreman-Mackey, D., Hogg, D. W., Lang, D., \& Goodman, J. 2013, PASP, 125, 306

Gaia Collaboration (Prusti, T., et al.) 2016, A\&A, 595, A1

Gaia Collaboration (Brown, A. G. A., et al.) 2018, A\&A, 616, A1

Gehrels, N. 1986, ApJ, 303, 336

Giesers, B., Dreizler, S., Husser, T.-O., et al. 2018, MNRAS, 475, L15

Giesers, B., Kamann, S., Dreizler, S., et al. 2019, A\&A, 632, A3

Gray, D. F. 1992, The Observation and Analysis of Stellar Photospheres (Cambridge: Cambridge University Press), 20 
Green, G. M., Schlafly, E. F., Finkbeiner, D., et al. 2018, MNRAS, 478, 651 Gu, W.-M., Mu, H.-J., Fu, J.-B., et al. 2019, ApJ, 872, L20

Güver, T., \& Özel, F. 2009, MNRAS, 400, 2050

Iben, I., Jr., Tutukov, A. V., \& Yungelson, L. R. 1995, ApJS, 100, 233

Irrgang, A., Geier, S., Kreuzer, S., Pelisoli, I., \& Heber, U. 2020, A\&A, 633, L5

Jayasinghe, T., Stanek, K. Z., Kochanek, C. S., et al. 2019, MNRAS, 486, 1907

Jones, D., Conroy, K. E., Horvat, M., et al. 2020, ApJS, 247, 63

King, A. R., \& Wijnands, R. 2006, MNRAS, 366, L31

Kochanek, C. S., Shappee, B. J., Stanek, K. Z., et al. 2017, PASP, 129, 104502

Lallement, R., Babusiaux, C., Vergely, J. L., et al. 2019, A\&A, 625, A135

Liu, J., Zhang, H., Howard, A. W., et al. 2019, Nature, 575, 618

Makarov, V. V., \& Tokovinin, A. 2019, AJ, 157, 136

McClintock, J. E., Haswell, C. A., Garcia, M. R., et al. 2001, ApJ, 555, 477

McClintock, J. E., \& Remillard, R. A. 2006, Black Hole Binaries (Cambridge: Cambridge University Press), 39, 157

Menou, K., Narayan, R., \& Lasota, J.-P. 1999, ApJ, 513, 811

Misra, D., Fragos, T., Tauris, T., Zapartas, E., \& Aguilera-Dena, D. R. 2020, A\&A, 642, A174

Olejak, A., Belczynski, K., Bulik, T., \& Sobolewska, M. 2020, A\&A, 638, A94

Pandey, J. C., \& Singh, K. P. 2012, MNRAS, 419, 1219

Podsiadlowski, P., Rappaport, S., \& Han, Z. 2003, MNRAS, 341, 385
Rivinius, T., Baade, D., Hadrava, P., Heida, M., \& Klement, R. 2020, A\&A, 637, L3

Safarzadeh, M., Toonen, S., \& Loeb, A. 2020, ApJ, 897, L29

Shao, Y., \& Li, X.-D. 2019, ApJ, 885, 151

Shappee, B. J., Prieto, J. L., Grupe, D., et al. 2014, ApJ, 788, 48

Shenar, T., Bodensteiner, J., Abdul-Masih, M., et al. 2020, A\&A, 639, L6

Steele, I. A., Smith, R. J., Rees, P. C., et al. 2004, in Ground-based Telescopes, eds. J. Oschmann, \& M. Jacobus, SPIE Conf. Ser., 5489, 679

Thompson, T. A., Kochanek, C. S., Stanek, K. Z., et al. 2019, Science, 366, 637

Thompson, T. A., Kochanek, C. S., Stanek, K. Z., et al. 2020, Science, 368, eaba4356

van den Heuvel, E. P. J., \& Tauris, T. M. 2020, Science, 368, eaba3282

Voges, W., Aschenbach, B., Boller, T., et al. 2000, IAU Circ., 7432, 3

Wade, R. A., \& Horne, K. 1988, ApJ, 324, 411

Walter, R., Lutovinov, A. A., Bozzo, E., \& Tsygankov, S. S. 2015, A\&ARv, 23, 2

Wiktorowicz, G., Lu, Y., Wyrzykowski, Ł., et al. 2020, ApJ, 905, 134

Wilms, J., Allen, A., \& McCray, R. 2000, ApJ, 542, 914

Yan, Z., \& Yu, W. 2015, ApJ, 805, 87

Yi, T., Sun, M., \& Gu, W.-M. 2019, ApJ, 886, 97

Yungelson, L. R., Lasota, J. P., Nelemans, G., et al. 2006, A\&A, 454, 559

Zheng, L.-L., Gu, W.-M., Yi, T., et al. 2019, AJ, 158, 179 